ISSN 1991-8631

Original Paper

http://indexmedicus.afro.who.int

\title{
Fragmentation d'un écosystème Littoral : cas de la baie de Loango au Congo-Brazzaville
}

\author{
Arsène LENGA ${ }^{1,2^{*}}$, Louzolo Enock BAGHAMBOULA ${ }^{1}$ et Joseph VOUIDIBIO ${ }^{2}$ \\ ${ }^{1}$ Laboratoire de Biécologie des Vertébrés et Invertébrés (LBEVI), Département de Biologie et Physiologie \\ Animale, Faculté des Sciences et Techniques, Université Marien NGOUABI, Brazzaville, Congo. \\ ${ }^{2}$ Groupe de Recherche sur la Biologie des Populations Animales, Faculté des Sciences et Techniques, \\ Université Marien NGOUABI, Brazzaville, Congo. \\ *Auteur correspondant ; E-mail : arsenelenga@yahoo.fr ; B.P. 1375 Brazzaville, Congo \\ Tél. (242) 5510244 ; (242)6637521
}

\section{RESUME}

L'action de l'homme sur la modification profonde et dans certains cas irréversibles de son milieu est étudiée dans une partie de la baie de Loango, à Matombi au Nord de Pointe-Noire. Nous avons par des études préliminaires, recensé différentes perturbations de cet écosystème littoral et analysé les pressions écologiques exercées par la modification des biotopes voisins. Les observations se sont déroulées du 07 au 21 septembre en 2007, et en 2008 du 13 juin au 10 août puis du 27 octobre au 09 décembre de la même année, à une fréquence d'une visite tous les deux jours, soit quatre visites par semaine et seize visites par mois. Les précipitations, l'abattage des arbres et des arbustes, l'élimination abusive de la strate herbacée à des fins touristiques et l'érosion marine sur le continent sont les principaux facteurs concourant à la perturbation du littoral de Matombi. Ce processus se traduit par une modification de la répartition des espèces animales et végétales, des habitats et microhabitats et stimule les possibilités adaptatives des espèces animales Invertébrés (comme les crustacés, certains hyménoptères, des Isoptères) mais aussi des Vertébrés (Oiseaux et certains petits mammifères).

(C) 2012 International Formulae Group. All rights reserved.

Mots clés: Dégradation, écosystèmes côtiers, Habitats, Biodiversité, tropical.

\section{INTRODUCTION}

La dégradation des écosystèmes est une des conséquences majeures de la surexploitation des milieux par l'homme. Le résultat est l'indisponibilité des ressources nécessaires à la survie des animaux. Dès l'aube du quaternaire, les hominiens commencèrent à modifier profondément les biocénoses continentales. Aucun écosystème depuis lors, quel que soit son étendu n'a pu échapper à l'action anthropique (Adams, 2004 ; Thomas et al., 2004 ; Chauveau, 2012). C'est ainsi que les écosystèmes terrestres, continentaux et marins ont largement été modifiés et font actuellement l'objet de nombreuses études pour leur conservation à travers la production et la conservation de la biodiversité (Ramade, 1994 ; Durand, 1999 ; Ramade, 2002 ; Neuenschwander et al., 2011). Les zones littorales revêtent des 
importances socio-économique, culturelle, environnementale et scientifique essentielles, dans la mesure où elles représentent un domaine hautement stratégique dans le fonctionnement d'un Etat (PNUE, 2000). Ainsi, les écosystèmes côtiers et la biodiversité de l'Afrique fournissent d'importants moyens de subsistance à des milliers de ménage. Les observations réalisées depuis deux décennies, montrent que les deux tiers de la population mondiale vivent à proximité des océans (Ramade, 2005). Dans le cas du littoral africain, on évalue actuellement à $38 \%$ le taux de côtes fortement menacées. En effet, les zones littorales sont exposées à diverses agressions qui réduisent l'espace utilisable et fragmentent les différents écosystèmes en présence (Elenga et al., 1992).

La surexploitation excessive des écosystèmes se traduit de nos jours par une diminution continue des habitats spécifiques aux milieux terrestres, aux eaux continentales et marines et partant, favorise la perturbation de la biodiversité animale et végétale (Anonyme, 2001; Frontier et Pichod-Viale, 1998 ; Ramade, 2005).

L'action de l'Homme est souvent à l'origine d'un enchaînement de fragmentation des écosystèmes actuellement en cours d'étude par de nombreux organismes tant nationaux qu'internationaux, à travers le réchauffement climatique dont l'influence est suspectée dans la baie de Loango à Matombi au Congo-Brazzaville.

Les travaux de Sitou (1991), Batchy et Locko (2003) et Batchy (2004) ont permis de quantifier le recul du trait de côte à plusieurs endroits du littoral congolais, cependant les pressions écologiques occasionnées par la modification des biotopes en général et de Matombi en particulier n'ont pas encore fait l'objet de sérieuses investigations. Dans le but de compléter les données acquises visant à terme, à la protection et la conservation d'un écosystème sensible comme celui de la baie de Loango, nous avons procédé à un recensement des différentes perturbations agissant sur le littoral, en évaluant l'importance de la fragmentation des écosystèmes littoraux sur la répartition des espèces animales et végétales. L'influence de la fragmentation des écosystèmes sur la modification des habitats et les différentes possibilités d'adaptation développées par les animaux sont également abordées ici.

\section{MATERIEL ET METHODES \\ Présentation du milieu d'étude}

Le milieu d'étude est situé à l'Ouest du Congo-Brazzaville, à environ $25 \mathrm{~km}$ au Nord de la capitale économique Pointe-Noire et au bord de l'océan atlantique. Le village matombi représente le centre du milieu d'étude, délimité entre les gorges de Diosso au Nord -Est et la baie de Loango au bord de l'océan atlantique. Le site d'étude s'étend entre $11^{\circ}, 10^{\circ}$ et $12^{\circ}$ de longitude Ouest et entre $4^{\circ}$ et $5^{\circ}$ de latitude Sud. Le climat est de type équatorial à saison alternée relativement humide. Le régime pluviométrique est caractérisé par deux saisons nettement tranchées : une saison des pluies d'octobre à mai et une saison sèche de juin à septembre (Batchy, 2001). Le volume des précipitations annuelles moyennes oscille autour de 1260 $\mathrm{mm}$ dans la région de Pointe-Noire.

Le substrat est un ensemble de sols ferralitiques développés sur des matériaux sableux ou sablo-argileux pauvres en base et très perméables. Les matériaux sont issus des formations géologiques de la série des cirques (Elenga et al., 1992).

Le milieu d'étude peut être subdivisé en quatre zones en rapport avec le village Matombi : Matombi urbain, Matombi village, Matombi littoral et Matombi-gorges de Diosso.

Matombi urbain se caractérise par la présence de nombreux restaurants, de chambres d'hôtes et de complexes spécifiques d'une zone urbanisée tels qu'un centre de santé intégré, un centre préscolaire, un terrain de football et de nombreux commerces.

Matombi village situé à la périphérie de la zone urbaine est réellement de type paysan avec de nombreuses constructions de fortune 
(cases, hangars..etc). Il y est pratiqué le fumage du poisson et l'extraction artisanale de minerais dans les carrières avoisinantes.

Matombi littoral se caractérise par plusieurs ports de pêche artisanaux d'où partent de nombreuses pirogues à moteurs.

Matombi-gorges de Diosso est situé en amont de matombi village et Matombi urbain. Ce site est occupé par une forêt galerie et quelques cours d'eau se jetant dans l'océan, dont le plus important est la rivière matombi. L'exploitation des essences forestières est orientée vers les sources d'énergie ou le bois de chauffe destiné aussi au fumage du poisson.

Pour des raisons pratiques, ce milieu d'étude a été subdivisé en sites nommés A, B, $\mathrm{C}$ et $\mathrm{D}$. Chaque site s'étend sur $500 \mathrm{~m}$ environ le long du littoral et présente des caractéristiques qui lui sont spécifiques.

\section{Site A}

Dans ce site prédomine une strate herbacée à Hyparrhenia diplandra, $H$. familiaris, Loudestia arundinace, Panicum congoense, Murdonnia simplex qui s'enfonce sur environ $30 \mathrm{~m}$ à l'intérieur du continent ; La strate arbustive est dominée par Nauclea latifolia, Hymenocardia acida, Annona arenaria reticulata, Cassippurea barteri, Imperata cylindrica, Aframomum sp, Aframomum latifolium et Syrtosermena senegalense ainsi que l'a observé Makany (1963, 1964).

\section{Site B}

Cette zone a été profondément modifiée par l'Homme en raison de nombreuses constructions qui y sont réalisées. La strate herbacée à Hyparrhenia sp. et Annona sp a été parfois remplacée par des essences d'ornementation comme Paspalum sp. Hibiscus sp. Bougainvilea sp. La strate arbustive est dominée par Cocos nucifera, Elaeis guinensis, Spiduim sp., Citrus cininsis et Bambusa vulgaris, ainsi que d'autres essences introduites par l'Homme. Les travaux de construction ont profondément fragilisé le sol de même que le désherbage et l'abattage des arbres. Cette zone est envahie par plusieurs visiteurs venus en villégiature le week-end.

\section{Site C}

Cette zone est marquée par la présence d'une station de pêche artisanale. On note une prédominance de la strate herbacée à $H$. diplandra qui s'enfonce sur environ $25 \mathrm{~m}$ à l'intérieur des terres malgré quelques constructions humaines (hangars, cases,...). La strate arbustive est marquée par la présence de quelques arbres fruitiers: Mangifera indica, Dacryodes edulis, Carica papaya, E. guinensis, Annona sp. et Eucalyptus sp.. Cette zone est traversée par un écosystème particulier : il s'agit de la rivière Matombi qui se jette directement dans l'océan Atlantique.

\section{Site D}

Il s'agit des gorges de Diosso. Le lambeau forestier le mieux conservé est en position rélictuelle, souvent appelé cirques bien qu'il s'agisse de ravins digités. La composition floristique de ces lambeaux forestiers, avec un mélange d'espèces à écologie diverse comme leur petite surface et leur discontinuité, montre à l'évidence que ce sont des restes très fortement anthropisés d'une forêt primitive probablement semicaducifoliée (Makany, 1963). Ce lambeau forestier abrite une biodiversité animale et végétale importante.

Le matériel ayant servi à l'étude des sites susmentionnés a été constitué de divers instruments d'écologie de terrain à savoir : des filets fauchoir, des filets à papillon, des boîtes à insectes, un appareil de berlèsetullgren pour la capture de la microfaune édaphique, des tamis, une paire de jumelles, un appareil photo numérique, un décamètre, un thermomètre, du matériel classique de laboratoire de zoologie, constitué essentiellement de pinces souples, et fines. Une loupe binoculaire de type Leica 2000 Zoom a permis l'observation des spécimens récoltés avant identification grâce aux clés de 
détermination de Caspari et al. (1961), Picker et al. (2004), ou aux indications morphoanatomiques fournies par d'autres auteurs (Aron et Grassé, 1966). Ceux-ci ont été conservés dans des bocaux de $10 \mathrm{~cm}^{3}$. De l'alcool à $70{ }^{\circ} \mathrm{C}$ et du formol à $30 \%$ ont été utilisés pour la conservation sans altération physique des animaux capturés.

Une étuve de type Memmert de capacité 400 ${ }^{\circ} \mathrm{C}$ a également été utilisée pour l'analyse des échantillons de sol.

La méthode de travail a consisté en la délimitation d'une zone d'étude de $2 \mathrm{~km}$ de long subdivisée en quatre sites présentés plus haut: Il s'agit des sites A, B et C dans le village de Matombi et le site D dans les gorges de Diosso. Chaque site a été fixé à 500 $\mathrm{m}$ de long, s'étendant le long du littoral et séparé du suivant d'un intervalle de $200 \mathrm{~m}$. Sur le continent, les observations ont été faites sur $40 \mathrm{~m}$ en profondeur à partir d'un point donné du littoral. Des repères ont été placés pour délimiter chaque site, aussi bien dans la zone du littoral qu'à l'intérieur du continent. Les visites sur le terrain se sont effectuées en trois phases sur deux années successives :

- La première phase ou phase de première approche effectuée en 2007 s'est étalée du 7 au 21 septembre soit 8 visites sur le terrain à raison d'une visite tous les deux jours de 9 heures à 17 heures dans chaque site. Cette phase a représenté une première évaluation des biotopes à étudier. Des séjours réalisés plus tard ont permis de préciser les observations.

- La deuxième phase beaucoup plus longue que la première s'est étalée du 13 juin au 10 août 2008 dans les mêmes sites. Au total, 28 visites ont été effectuées sur le terrain à raison d'une visite tous les deux jours de 09 heures à 17 heures, soit 3 ou 4 visites par semaine et 14 visites par mois.

- La troisième phase s'est étalée du 27 octobre au 09 décembre 2008 dans les mêmes sites à raison d'une visite tous les deux jours aux heures susmentionnées. Au cours de cette phase, 23 visites ont été effectuées sur le terrain soit 3 ou 4 visites par semaine.
Dans les trois sites, le travail consistait à :

- Estimer l'importance de la couverture végétale. Cette estimation portait essentiellement sur le nombre et les espèces arbustives et herbacées rencontrées ;

- Estimer le nombre et les espèces animales Invertébrés et Vertébrés présentes au cours de la période d'étude ;

- Noter les différents horizons mis à nu ainsi que leur variation au niveau du littoral ;

- Estimer la dénivellation (hauteurs de la falaise par rapport au niveau de l'océan) à chaque visite ;

- Estimer le nombre et les types d'habitats et de micro-habitats dans les différents sites littoraux.

Pour ce faire:

- Les arbres marqués ont été dénombrés ;

- La superficie dégradée au cours des éboulements a été estimée ;

- La capture des insectes a été réalisée par fauchage dans des zones délimitées. Les insectes déjà déterminés ont été relâchés. L'observation des spécimens ramenés au laboratoire a ensuite été réalisée afin d'en déterminer les positions systématiques, selon les clés indiquées par d'autres auteurs (Grassé, 1976 ; Grassé, 1996 ; Baker, 1999).

- les hauteurs relatives de la falaise étaient évaluées et les habitats et micro-habitats ont été déterminés et comptés.

- Les différents horizons mis à nu ainsi que leurs variations au niveau du littoral étaient évalués. Dans ce dernier cas, une étude morphoscopique et granulométrique a été réalisée après séchage de l'échantillon et son pesage avant tamisage à l'eau (tamis de 20 $\mu \mathrm{m})$. Après séparation de la fraction fine et de la fraction sableuse, une étude granulométrique a pu être réalisée.

Des repères ont été placés à 2,50 $\mathrm{m}$ de la falaise pendant une heure et demie et le niveau du ressac, l'ampleur des éboulements, ainsi que la hauteur de la falaise ont été déterminés. 


\section{Analyses des données}

Les variations de la superficie des zones fragmentées ont été analysées selon les notions de variance, d'écart-type et de coefficient de corrélation développées par Masiéri (1996).

\section{RESULTATS}

Estimation de la variation de la strate arbustive dans le site $A$

Comme l'indique la Figure 1, nous avons observé une baisse graduelle du nombre d'arbres en fonction du temps. Ainsi, 10\% des espèces de $A$. arenaria ont été éliminés entre septembre 2007 et juillet 2008. Ce même constat a été fait pour Eucalyptus sp. Pour ces deux espèces, les arbres ont été, soit abattus par l'Homme, soit emportés par l'effondrement du littoral. Dans le cas de $E$. guinensis et C. nucifera, $10 \%$ et $20 \%$ respectivement de ces végétaux ont été éliminés du littoral à la suite de l'effondrement de celui-ci entre septembre 2007 et décembre 2008 dans le premier cas et entre juillet 2008 et décembre 2008 dans le deuxième cas.

Estimation de la variation de la strate arbustive dans le site $B$

Les résultats obtenus montrent que $10 \%$ de l'espèce $A$. arenaria ont été perdus par abattage entre septembre 2007 et juillet 2009. Par ailleurs, les Eucalyptus observés ont été soit abattus (7\%), soit éliminés lors de l'effondrement du littoral (3\%) (Figure 2). Dans le cas de E. guinensis, l'effondrement du littoral (Photo1) a été la cause principale de son élimination (4\%). De même, $10 \%$ des $C$. nucifera ont été éliminés par l'effondrement du littoral entre septembre 2007 et juillet 2008.

Dans ce site, $M$. indica et Carica papaya ont été observés et si $6 \%$ des premiers ont été éliminés entre septembre 2007 et juillet 2008, 15\% des seconds l'ont été à la même période. La perte de ces derniers étant augmentée considérablement à $31 \%$ entre juillet et décembre 2008.
Estimation de la perte de la strate herbacée

Les variations de la surface herbacée dégradée en 2007 sont très importantes dans les sites A et C. Si le site B est resté relativement stable au cours de la période d'étude, les sites $\mathrm{A}$ et $\mathrm{C}$ ont fortement varié respectivement de $39,31 \pm 9,7 \mathrm{~m}^{2}$ et $29,72 \pm$ $7,96 \mathrm{~m}^{2}$ durant la période expérimentale (Tableau 1).

Lorsque les études ont été réalisées en période sèche en 2008, les observations ont indiqué ( Tableau 2) que la strate dégradée est de $31,16 \mathrm{~m}^{2}$ dans le site $\mathrm{A}$, de $22,04 \mathrm{~m}^{2}$ dans le site B et enfin, de $36,71 \mathrm{~m}^{2}$ dans le site C. La comparaison des coefficients de variation a montré une corrélation positive $(+0,27)$ entre les sites $\mathrm{A}$ et $B$ et négative $(-0,26)$ entre les sites $A$ et $C$, ceci indiquant bien l'hétérogénéité des éboulements au niveau des sites étudiés.

Lorsque les études ont été réalisées au cours de la saison des pluies, les résultats ont indiqué (Tableau 3) une plus grande variation de la surface dégradée comparée à la période sèche. En effet, un total de $135,89 \mathrm{~m}^{2}$ pour le site $A, 101,90 \mathrm{~m}^{2}$ pour le site $B$ et $168,65 \mathrm{~m}^{2}$ pour le site $\mathrm{C}$ ont été obtenus. Dans ces trois cas, la variation de la surface dégradée a évolué de façon identique. En effet, les coefficients de variation sont positifs dans les trois cas, respectivement de $+0,95$ pour les sites $\mathrm{A}$ et $\mathrm{B}$, de $+0,85$ pour les sites $\mathrm{A}$ et $\mathrm{C}$ puis de $+0,79$ pour les sites B et C. Les variations sont relativement du même ordre quel que soit le site.

La Figure 3 montre la variation de la strate arbustive dans le site $\mathrm{C}$.

\section{Estimation de la biodiversité des Vertébrés et des Invertébrés dans les sites étudiés \\ Plusieurs groupes de Vertébrés et} Invertébrés ont été recensés au cours de notre étude dans chaque site durant les années 2007 et 2008. Dans le cas des Vertébrés, comme l'indique la Figure 4, divers Oiseaux et Batraciens ont été observés.

Deux espèces, à savoir Corvus albus Müller (Passériforme: Corvidae) et Passer 
griseus Vieillot (Passériforme: Passeridae), ont été observées (Figures 4 et 5). Les taux obtenus au cours des années 2007 et 2008 pour la même saison sèche indiquent une augmentation du nombre d'individus, notamment pour les oiseaux dont les taux sont passés de 26 à 34\% dans nos conditions expérimentales. Cette remarque est valable pour les Lacertiliens dont le taux est passé de 13 à $41 \%$ aussi bien dans le site A que dans le site B. Dans ce dernier site, nous avons noté l'apparition de Canis sp tandis que les rongeurs et les Batraciens n'y ont pas été observés. Dans le site C, ce même schéma est observé, c'est-à-dire que les oiseaux (C. albus et $P$. griseus) ainsi que Agama agama savattieri Rochebrune, (Squamate : Aganidae) ont été les animaux les plus rencontrés. En outre, la présence peu significative de Canis familiaris domesticus Linnaeus (Carnivore: Canidae) a été notée. Il ressort de cette étude que les taux de Vertébrés observés ont augmenté d'une année à l'autre au cours de la saison sèche dans les sites $\mathrm{A}, \mathrm{B}$ et $\mathrm{C}$ et remarquablement en période de pluies de l'année 2008.

\section{Estimation de la variation des Invertébrés au cours des années 2007 et 2008 dans les différents sites}

Plusieurs groupes d'Invertébrés ont été capturés, observés puis relâchés par la suite. Les groupes zoologiques les plus significatifs recensés sont les Arthropodes dont les Crustacés en zone intertidale, puis les Insectes (Orthoptères, Hyménoptères, Lépidoptères, Odonatoptères) sur le continent, dans les zones d'étude. Les Diptères, les Hétéroptères et les Coléoptères ont également été observés, cependant, en raison de leur taux peu important, ils ne sont pas reportés ici.

Dans les trois sites A, B et C, la baisse globale des ordres pré-cités a été effective, les Hyménoptères, Orthoptères, Lépidoptères et Odonatoptères ont vu leur taux baisser au cours des deux saisons sèches de $12 \%, 14 \%$, $14 \%$ et $12 \%$ respectivement pour le site $\mathrm{A}$, de
$9,8,9$ et $12 \%$ pour le site $\mathrm{B}$, de $19,11,5$ et $7 \%$ pour le site $\mathrm{C}$ (Figures 6, 7, 8 et 9 ).

Pour le cas spécifique des crustacés et notamment les crabes observés sur la zone intertidale, les taux n'ont pas changé fondamentalement, variant entre 1 et $2 \%$ au cours des deux saisons sèches des années 2007 et 2008.

\section{Variation de la dénivellation du littoral au cours des années 2007 et 2008}

La hauteur de la falaise s'est considérablement modifiée dans chaque site au cours des deux années d'études. Et, cette variation s'est essentiellement caractérisée par une augmentation de la hauteur des falaises. Comme l'indiquent les Figures 10 et 11 en période sèche et en 2007 , la hauteur moyenne de la falaise a beaucoup variée, dans les sites $\mathrm{A}, \mathrm{B}$ et $\mathrm{C}$; elle est respectivement de $6,62 \pm 1,36 \mathrm{~m}, 6,20 \pm 0,11 \mathrm{~m}$ et $5,32 \pm 1,15 \mathrm{~m}$. Seul le site A a connu une variation importante de la hauteur de sa falaise. Par contre, en période de pluies, une variation moins importante de la dénivellation des falaises a été constatée (Figure 11) avec 7,70 $\pm 0,44 \mathrm{~m}$ pour le site $\mathrm{A}, 7,03 \pm 0,18 \mathrm{~m}$ pour le site $\mathrm{B}$ et $5,71 \pm 0,42 \mathrm{~m}$ pour le site $\mathrm{C}$.

\section{Estimation des habitats et micro habitats dans la zone d'étude}

Les différents habitats ont été principalement représentés par des terriers pour le crabe Ocypode cursor Linnaeus (Décapode: Ocypodidae), des galeries pour les Hyménoptères (Formicoidea) et les Isoptères, des trous dans la falaise pour les Oiseaux, et des passages ou pistes pour les petits Mammifères comme Civettictis civetta Schreber (Carnivore: Viverridae). Leur répartition comme l'indique les Tableaux 4 et 5 , ne montre aucune modification importante quelles que soient les perturbations se produisant sur le littoral au cours de deux années consécutives, les habitats et/ou micro habitats se redistribuent aussi rapidement qu'ils sont détruits par les éboulements. Les nids d'oiseaux, les terriers de crabes et les trous d'abeilles ont été notés comme les plus sensibles à la destruction du biotope, en raison de leur nombre relativement plus faible l'année suivante. Les animaux ont été 
retrouvés dans leurs habitats durant toute la période d'étude. Aucune désertion définitive d'habitats n'a été observée. Pour les galeries apparentes des termites exposées à différentes intempéries (ensoleillement excessif, averses violentes et multiples, érosion..etc), le nombre observé est plus élevé l'année suivante.

\section{Détermination des différents horizons dans la zone d'étude}

L'étude de la structure géologique de la falaise à Matombi à partir des échantillons de sol récoltés dans les sites $\mathrm{A}, \mathrm{B}$ et $\mathrm{C}$, indique une présence importante de sable grossier et de sable fin. Dans le site A, les prélèvements effectués à une profondeur totale de $3,50 \mathrm{~m}$ ont indiqué la présence de sable (jaune, gris, jaunâtre argileux). Le sable occupe une profondeur de $2,40 \mathrm{~m}$, tandis que les limons et les argiles rouges se répartissent sur $1,10 \mathrm{~m}$. Environ $70 \%$ du substrat ont été représentés par du sable et $30 \%$ par du limon et des argiles. Cependant, le sable et les argiles ont été trouvés mélangés à partir de $2 \mathrm{~m}$ de profondeur. Ces horizons ont porté une litière composée de feuilles mortes et de nombreux détritus végétaux. Aucun substrat caillouteux ni graveleux n'a été trouvé dans ce site. Dans le site $\mathrm{B}$, l'étude réalisée sur une profondeur de falaise de $2 \mathrm{~m}$ a montré que les sables sont répartis sur $1,23 \mathrm{~m}$ soit $61,5 \%$ et les limons et les argiles, sur $0,77 \mathrm{~m}$, soit $29,50 \%$ de la profondeur totale. L'analyse granulométrique a montré une prédominance de sables grossiers et de sables fins. Comme précédemment, les graviers et les cailloux n'ont pas été trouvés dans ce site. Le site $\mathrm{C}$ a montré les mêmes caractéristiques granulométriques que les sites précédents. Le sol est ainsi constitué essentiellement de matériaux sableux ou sablo-argileux facilement emportés par l'érosion. En outre, le sable a été observé occupant la partie supérieure moins humide, tandis que les limons et les argiles ont été trouvés dans les strates beaucoup plus humides, lesquelles sont exposées aux éboulements.

Tableau 1: Variation de la surface herbacée dégradée en 2007.

\begin{tabular}{lccc}
\hline \multirow{2}{*}{ Période d'observation } & \multicolumn{3}{c}{ Sites et surface dégradée en $\mathbf{~ m}^{\mathbf{2}}$} \\
\cline { 2 - 4 } & $\mathbf{A}$ & $\mathbf{B}$ & $\mathbf{C}$ \\
\hline J1 & 39,31 & 24,36 & 29,72 \\
J5 & 22,03 & 0 & 25,09 \\
J10 & 20,61 & 0 & 21,76 \\
TOTAL & 90,95 & 24,36 & 76,57 \\
\hline
\end{tabular}

Tableau 2: variation de la surface herbacée dégradée en 2008 (Période sèche).

\begin{tabular}{lccc}
\hline Période d'observation & \multicolumn{3}{c}{ Sites et surface dégradée en $\mathbf{~ m}^{\mathbf{2}}$} \\
\cline { 2 - 4 } & $\mathbf{A}$ & $\mathbf{B}$ & $\mathbf{C}$ \\
\hline J1 & 30,31 & 0 & 41,48 \\
J15 & 27,67 & 27,67 & 36,78 \\
J30 & 32,40 & 30,13 & 30,64 \\
J45 & 34,29 & 30,27 & 37,97 \\
TOTAL & 146,87 & 88,16 & 146,87 \\
\hline
\end{tabular}


Tableau 3: variation de la surface herbacée dégradée en 2008 (Période de pluies).

\begin{tabular}{lccc}
\hline \multirow{2}{*}{ Période d'observation } & \multicolumn{3}{c}{ Sites et surface dégradée en $\mathbf{~ m}^{\mathbf{2}}$} \\
\cline { 2 - 4 } & $\mathbf{A}$ & $\mathbf{B}$ & $\mathbf{C}$ \\
\hline J1 & 39,16 & 28,08 & 41,37 \\
J15 & 26,56 & 23,54 & 44,22 \\
J30 & 25,16 & 18,84 & 36,89 \\
J45 & 45,01 & 31,44 & 46,17 \\
TOTAL & 135,89 & 101,90 & 168,65 \\
\hline
\end{tabular}

Tableau 4: Nombre et types d'habitats dans chaque site en 2007 (période sèche).

\begin{tabular}{|c|c|c|c|c|}
\hline \multirow[b]{2}{*}{ Sites } & \multicolumn{4}{|c|}{ Types et nombre d'habitats } \\
\hline & $\begin{array}{l}\text { Trous/nids } \\
\text { d'Oiseaux }\end{array}$ & $\begin{array}{c}\text { Galeries } \\
\text { apparentes } \\
\text { des Isoptères }\end{array}$ & $\begin{array}{c}\text { Terriers de } \\
\text { Crabes }\end{array}$ & Trous d'Abeilles \\
\hline $\mathrm{A}$ & 07 & 05 & 189 & 29 \\
\hline B & 11 & 13 & 101 & 18 \\
\hline $\mathrm{C}$ & 20 & 18 & 96 & 0 \\
\hline
\end{tabular}

Tableau 5: Nombre et types d'habitats dans chaque site en 2008 (période sèche).

\begin{tabular}{ccccc}
\hline Sites & \multicolumn{4}{c}{ Types et nombres d'habitats } \\
\cline { 2 - 5 } & Trous/nids d'Oiseaux & $\begin{array}{c}\text { Galeries } \\
\text { apparentes des } \\
\text { Isoptères }\end{array}$ & $\begin{array}{c}\text { Terriers de } \\
\text { Crabes }\end{array}$ & Trous d'Abeilles \\
\hline A & 05 & 16 & 172 & 21 \\
B & 11 & 18 & 98 & 12 \\
C & 15 & 24 & 88 & 0 \\
\hline
\end{tabular}




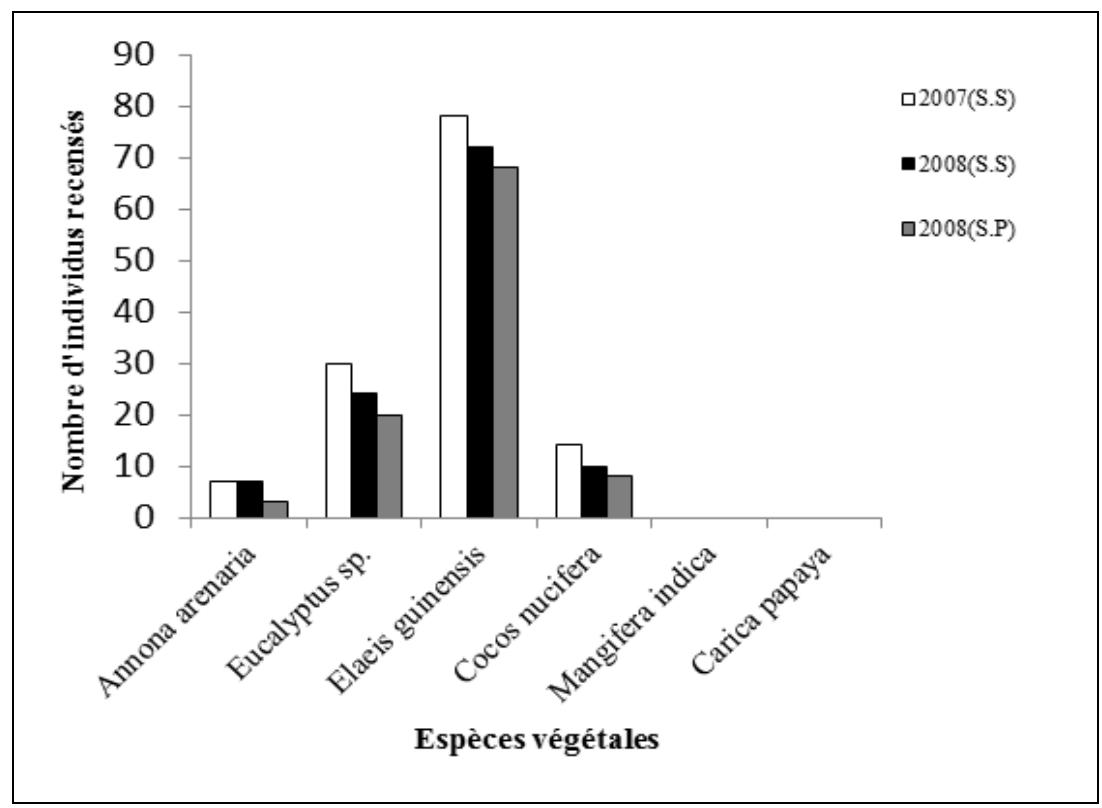

Figure 2: Variation de la strate arbustive dans le site B.

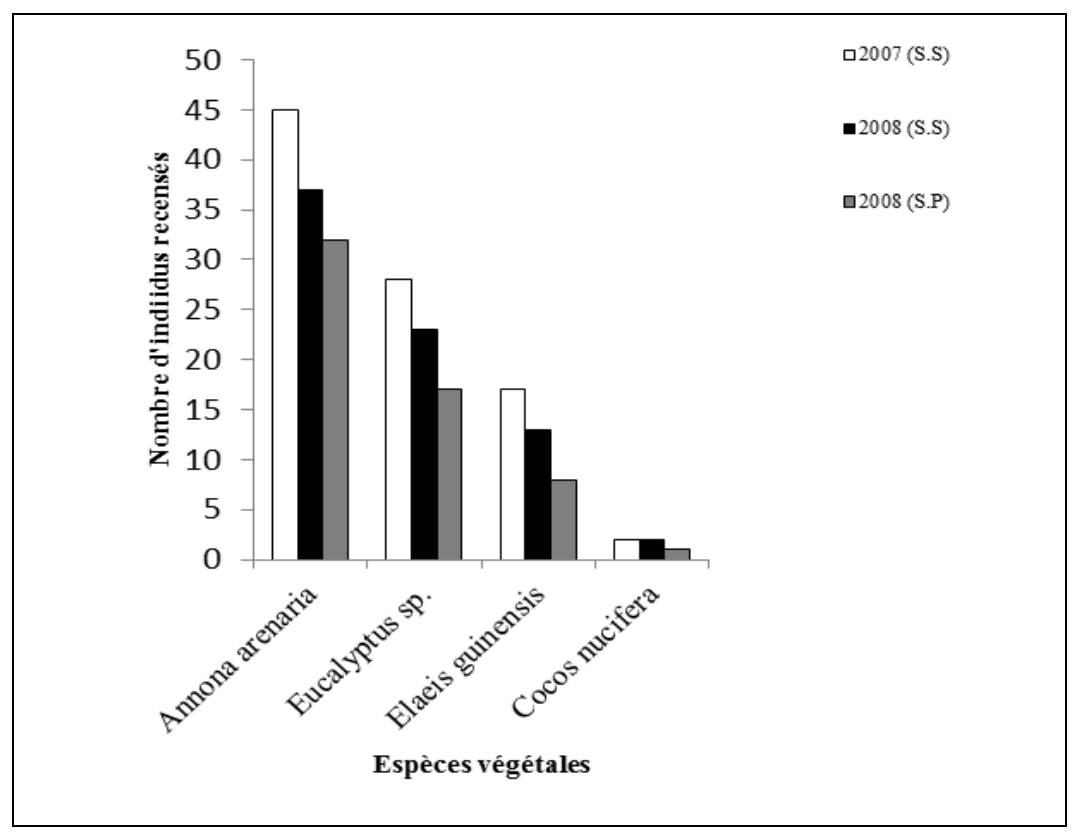

Figure 3: Variation de la strate arbustive dans le site C. 
A. LENGA et al. / Int. J. Biol. Chem. Sci. 6(5): 2113-2130, 2012

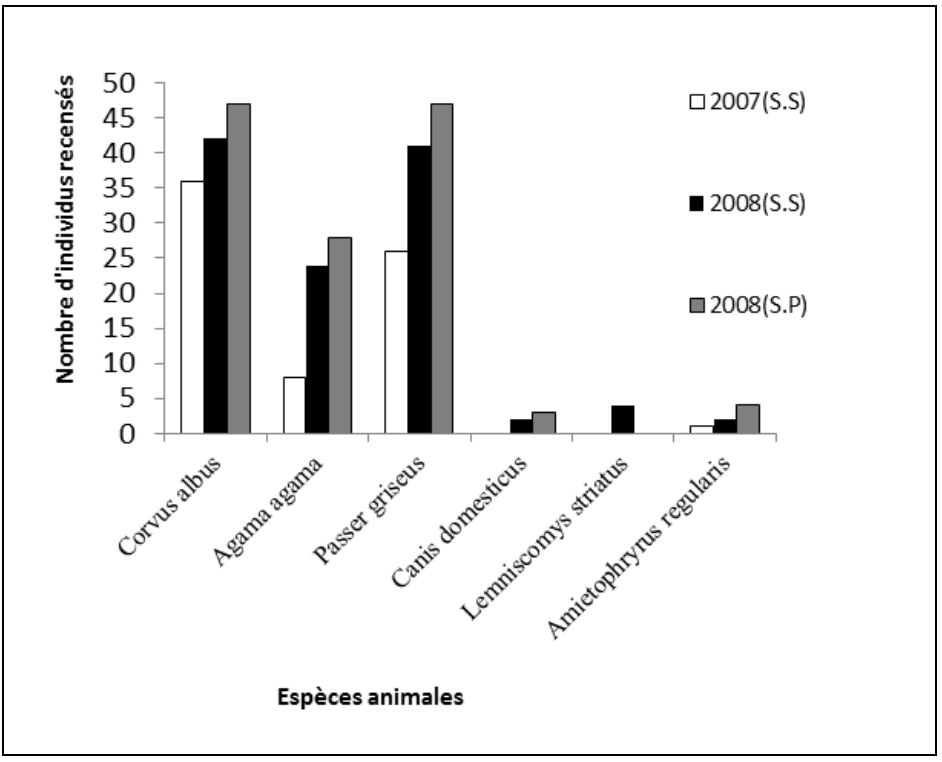

Figure 4 : Représentation de Vertébrés recensés dans le site A.

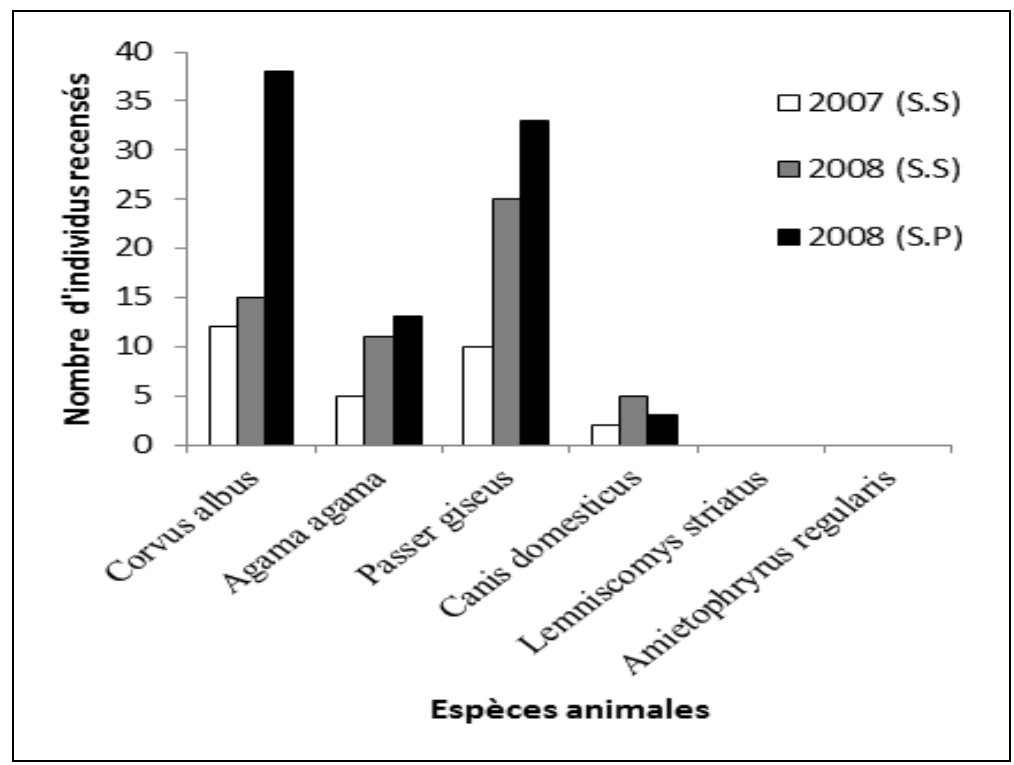

Figure 5: Représentation de Vertébrés recensés dans le site B. 
A. LENGA et al. / Int. J. Biol. Chem. Sci. 6(5): 2113-2130, 2012

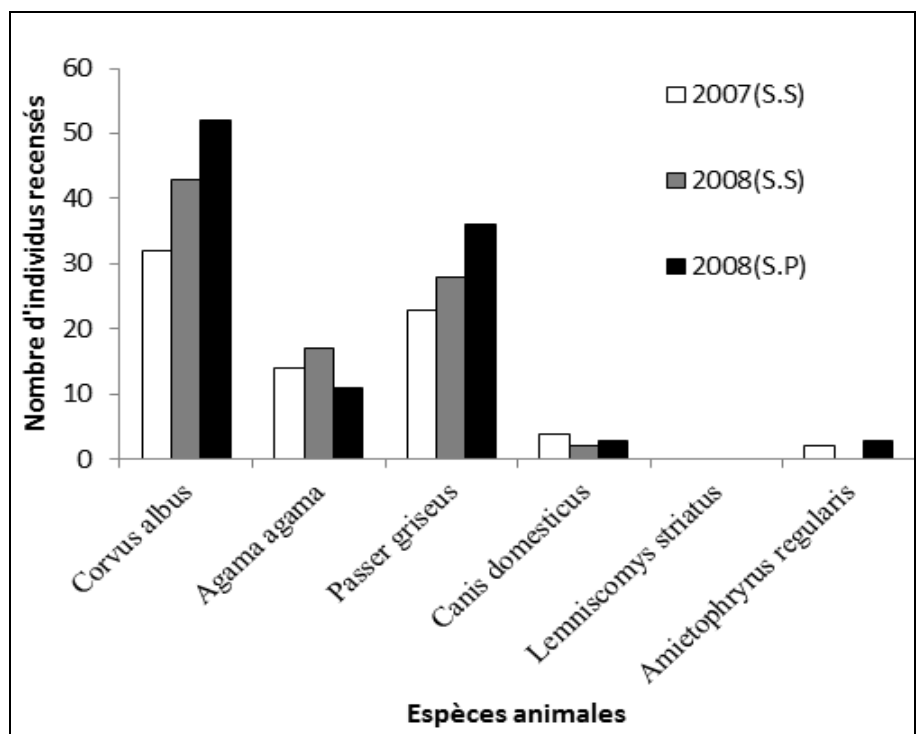

Figure 6 : Représentation de Vertébrés recensés dans le site C.

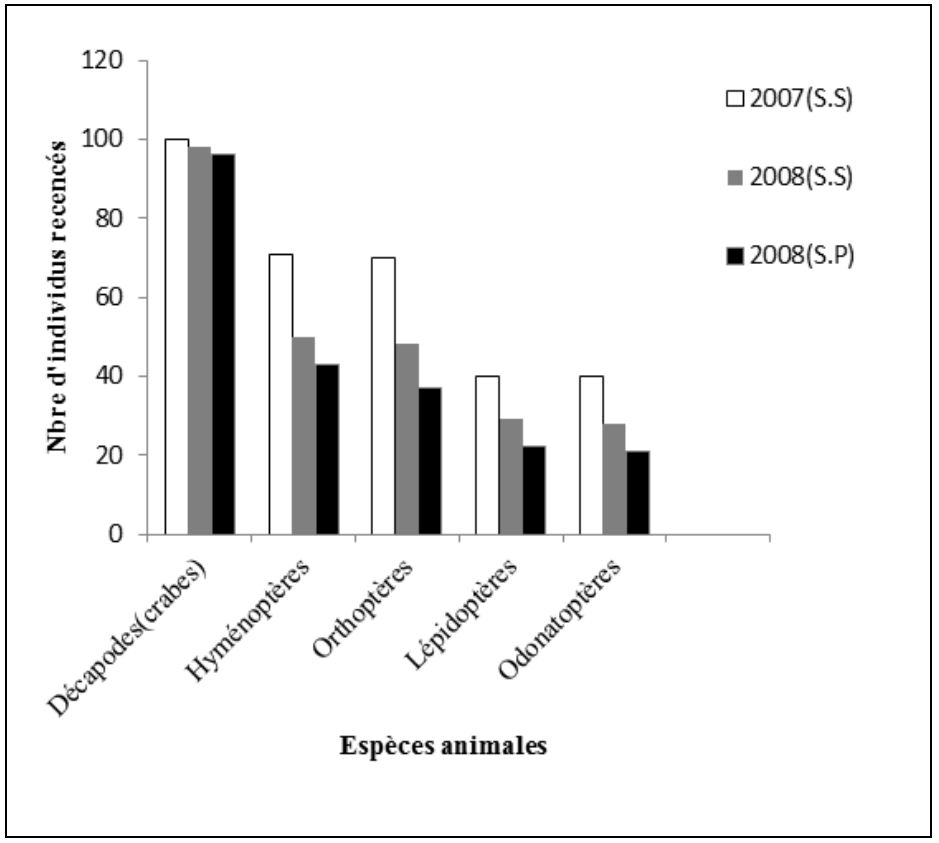

Figure 7 : Représentation des Invertébrés dans le site A. 
A. LENGA et al. / Int. J. Biol. Chem. Sci. 6(5): 2113-2130, 2012

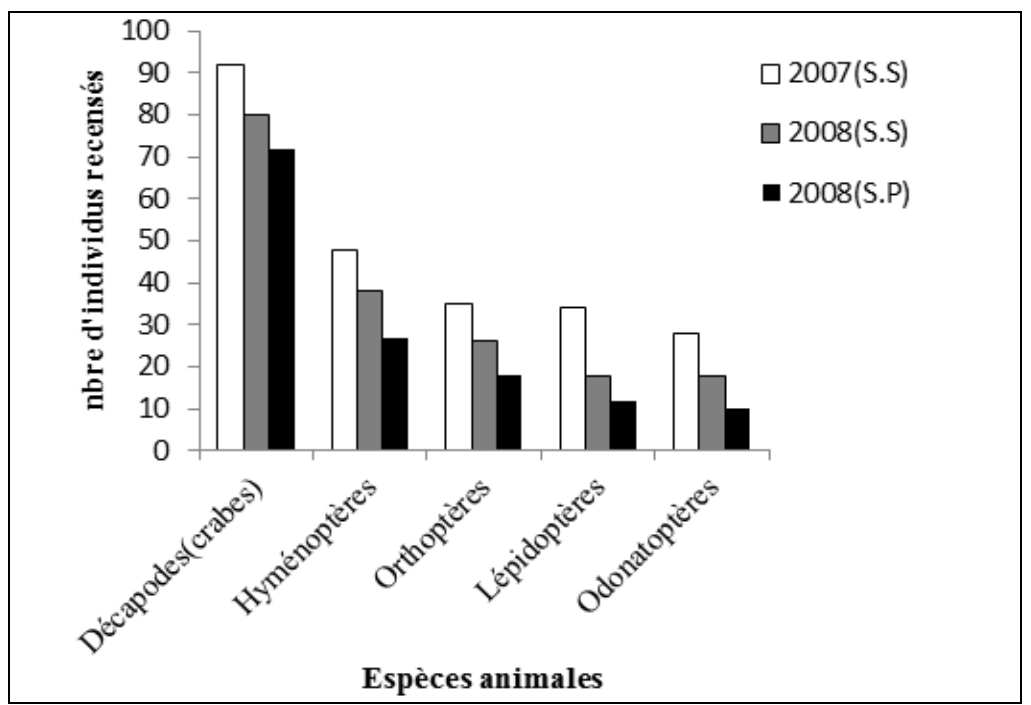

Figure 8: Représentation des Invertébrés dans le site B.

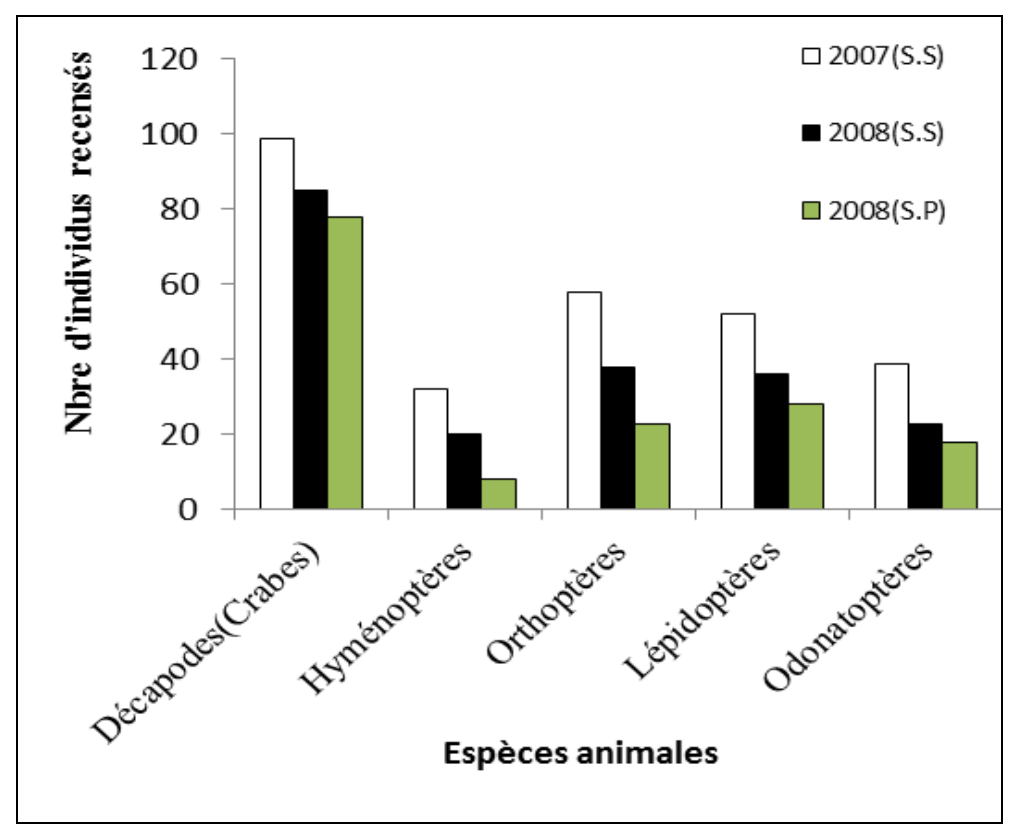

Figure 9: Variation des Invertébrés dans le site C. 


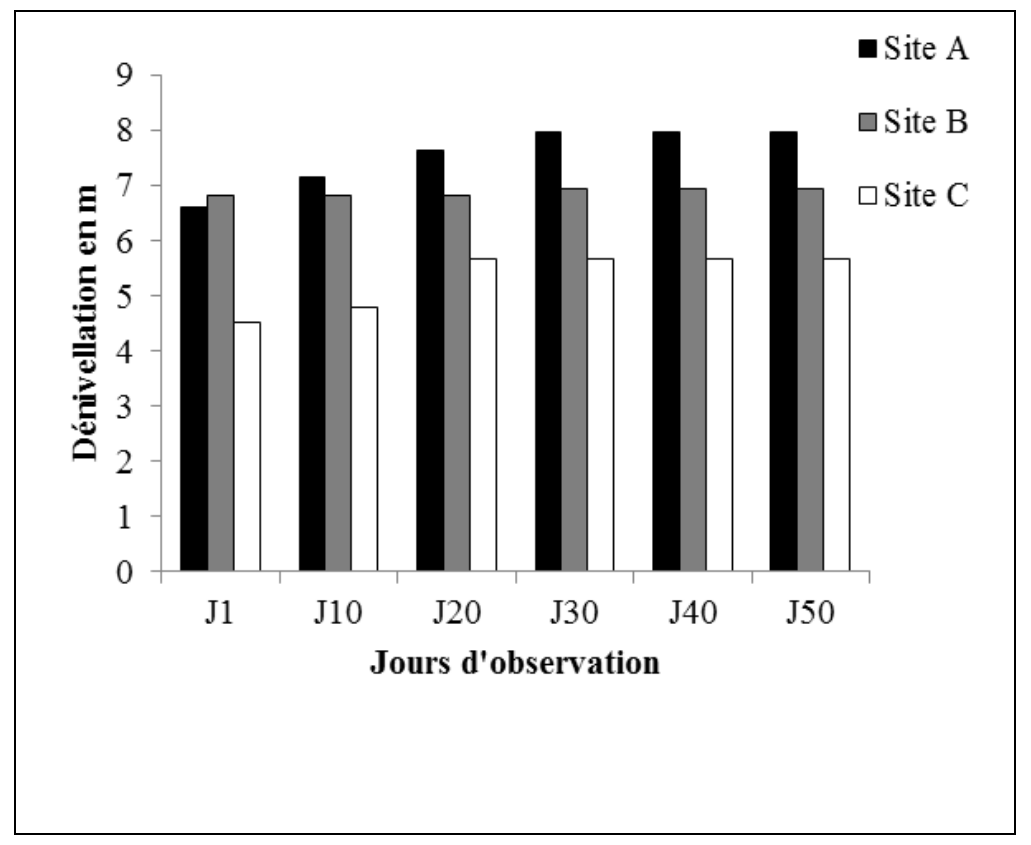

Figure 10: Variation de la dénivellation dans chaque site au cours de l'année 2008 (période sèche).

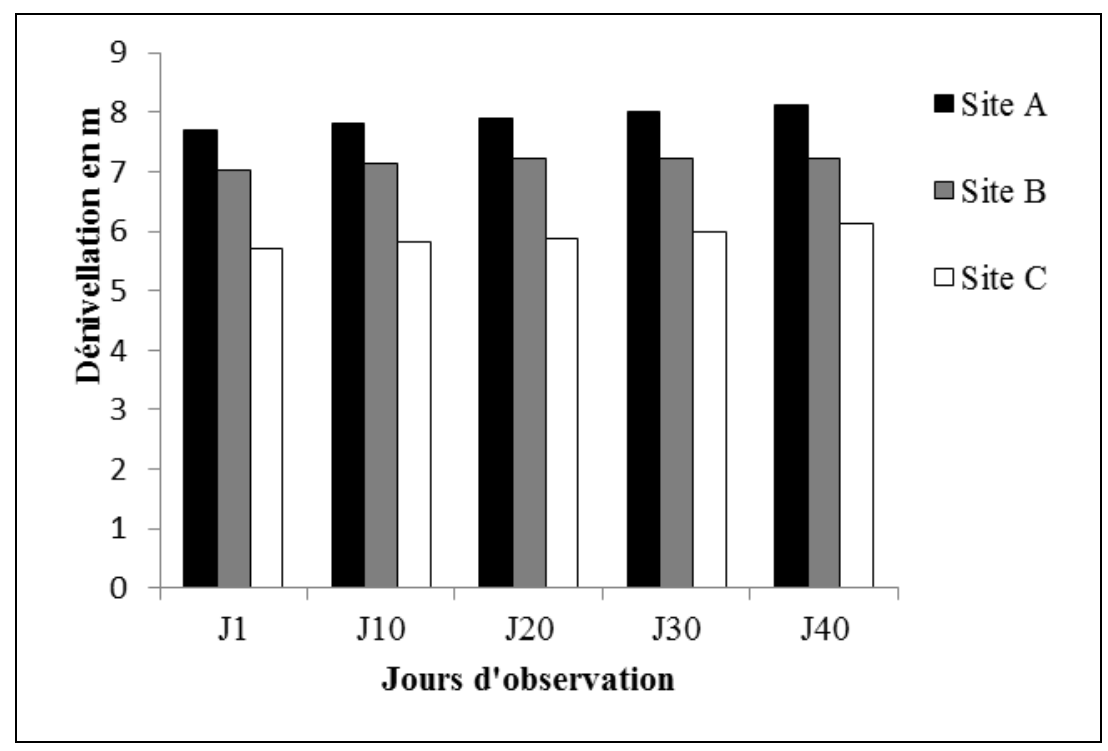

Figure 11: Variation de la dénivellation dans chaque site au cours de l'année 2008 (période pluvieuse). 


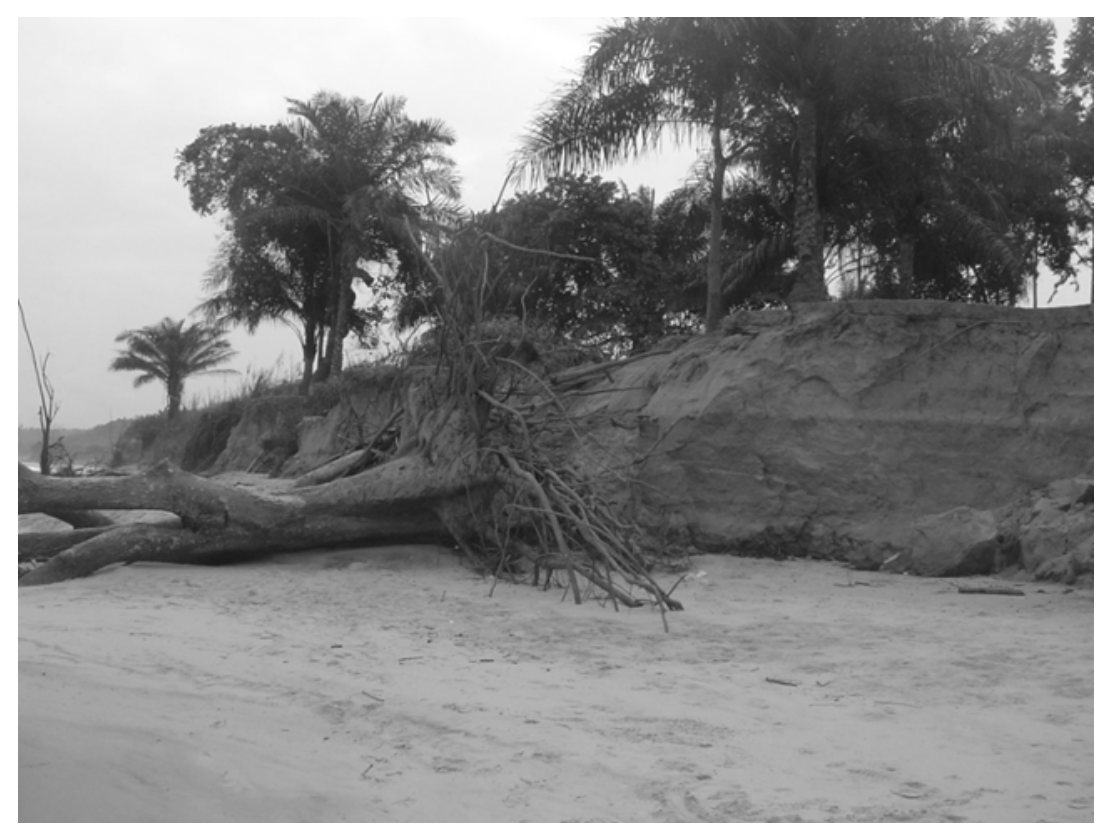

Photo 1: Effondrement du littoral à Matombi.

\section{DISCUSSION}

De nombreuses fragmentations écologiques d'origine anthropiques, à travers la construction des autoroutes ou des lignes à haute tension, ont été jugées responsables de la modification des habitats de certains animaux, avec comme résultat, la baisse de leur possibilité de reproduction (Fischer et Lindenmayer, 2007). Dans la même acception, la dégradation anthropique des écosystèmes continentaux ou littoraux est à l'origine d'une amplification des phénomènes d'érosion et de perturbation des cycles de vie de nombreux animaux. Le morcellement des habitats de ceux-ci a été désigné comme responsable de la diminution de la biodiversité animale et végétale dans plusieurs pays européens (Ramade, 2005). Nos résultats, qui complètent ceux obtenus par d'autres auteurs (Sitou, 1991 ; Batchy, 2004), indiquent une dégradation progressive et continue de la strate arbustive (Annona sp., E. guinensis, Eucalyptus sp., C. nucifera..etc) et de la strate herbacée (I. cylindrica, A. latifolium, Aframomum sp., Hyparrhenia sp...). Il est probable que les fortes érosions du littoral, provenant de l'avancée de l'océan sur le littoral de la baie de Loango, mais aussi des précipitations dont les eaux de ruissellement sont orientées par les constructions anarchiques humaines, le déboisement et l'important désherbage, soient à l'origine d'importants éboulements entraînant une partie de la falaise. Le recul du trait de côte observé lors de travaux précédents, a été corrélé au cours de notre étude avec les différentes activités anthropiques, mais aussi l'influence des variations de l'environnement de façon naturelle. La forte concentration de la population humaine dans les sites $\mathrm{B}$ et $\mathrm{C}$, notamment lors d'afflux des populations en villégiature dans la zone d'étude, semble être à l'origine de l'augmentation de la perte de nombreuses espèces végétales telles que $M$. indica, Eucalyptus sp., Annona sp. ..etc. A ce propos, nous envisageons des recherches portant sur l'influence des processus de respiration et de photosynthèse, à travers une étude approfondie de l'importance du cycle du carbone (variation du $\mathrm{CO}_{2}$ atmosphérique notamment) ou d'autres éléments biogènes, dans les phénomènes de morcellement des 
écosystèmes littoraux ou continentaux. Les moyens de lutte contre les éboulements utilisés par les populations sont obsolètes et peu efficaces. En effet, la construction de berges de béton bien que contribuant au ralentissement des phénomènes d'érosions, ne permet pas à moyen et long terme d'éviter l'effondrement du littoral. Un reboisement à partir d'espèces au système racinaire profond est souhaitable. Par ailleurs, de nombreux cadavres d'animaux abandonnés sur les plages par les pêcheurs, pourraient être à l'origine de la forte concentration des Oiseaux (C. albus, $P$. griseus), ou encore des Reptiles ( $A$. agama). Les restes de pêche dans les ports artisanaux représentent une source alimentaire importante pour des Vertébrés dont le cycle de vie en est tributaire. A ce propos, nous envisageons des études supplémentaires afin de préciser l'importance de la quantité et la qualité des cadavres d'animaux ainsi abandonnés, et les raisons de la répulsion humaine pour les espèces animales abandonnées dans les ports. Dans les zones où $C$. $f$. domesticus a été rencontrée, cette espèce inféodée à l'homme, est un symbole de la présence de celui-ci. Par contre, les conditions du milieu seraient peu favorables au Batracien Amietophryrus regularis Reuss (Anoure : Bufonidae). Cette espèce n'a pas été trouvée en nombre important dans les différents sites. Il est probable que les embruns salés mêlés au vent côtier et à la savane peu humide ne favorisent pas son inféodation au milieu. La diminution considérable de la strate herbacée mais aussi de la strate arbustive suivie de la mise à feu de celle-ci au cours de la saison sèche, pourraient expliquer la diminution de Passer griseus qui ne trouverait plus assez de ressources pour la régulation de son cycle de vie. La perte de la strate herbacée et arbustive est responsable de la diminution de nombreux animaux notamment chez les Orthoptères d'une année à l'autre. Il faut y ajouter la prédation exercée par les oiseaux sur les populations d'Arthropodes (Blondel, 1995). En effet, les Arthropodes et les Oiseaux se succédant dans les chaînes trophiques, on peut comprendre les baisses des taux d'insectes se produisant parallèlement à l'arrivée dans le milieu, de nombreux oiseaux attirés prioritairement par les restes de produits de la pêche. Comme l'ont suggéré de nombreux auteurs, la fragmentation des habitats a sans doute conduit à une diminution de la taille des populations par suite de la baisse des surfaces du milieu favorable (Souza et Brown, 1994 ; Dajoz, 2000 ; Kerth et Melber, 2009). En effet, les résultats obtenus par ces auteurs ont prouvé que les fragments de surface restant d'une fragmentation sont généralement insuffisants pour assurer la conservation optimale de la faune. La survie des populations dont les effectifs sont réduits devient aléatoire et les possibilités d'émigration sont faibles et pourraient empêcher la colonisation d'autres milieux favorables (Chambon, 1993). Il est possible que la fragmentation de l'habitat ai pu accroître les affaiblissements génétiques des populations, consécutives d'une part, à leur adaptation aléatoire à un changement des conditions environnementales, à la diminution de la longévité des individus par suite de la propagation des défectuosités physiologiques (Blondel, 1995), ou encore à la sensibilité aux pathologies nouvelles d'autre part (Grassé, 1976); ainsi, la perturbation des micro habitats ou habitats pourrait être responsable de la diminution des effectifs d'Invertébrés constatée lors de notre étude (Krues et Tscharntke, 1994; Suarez et al., 1998). En outre, les travaux réalisés à ce sujet indiquent que les relations interspécifiques (parasitisme, compétition, prédation...etc) peuvent en être affectées. Cependant, Levejoy et al. (1986), mais aussi Wood et Gillman (1998), ont montré que le nombre des espèces d'une zone soumise à perturbation peut aussi s'élever lors de la fragmentation des habitats. Ces auteurs ont en outre montré que les habitats perturbés renferment plus d'espèces que les habitats non perturbés. L'hétérogénéité du milieu a été avancée pour expliquer cette possibilité. En effet, on peut 
comprendre que les papillons de la lisière et de la canopée, par exemple, qui recherchent des milieux bien éclairés, augmentent d'abondance avec la fragmentation de la forêt. D'autres auteurs ont évoqué les espèces écotoniales, lesquelles sont souvent ubiquistes et peuvent donc être favorisées dans le contexte d'un morcellement des écosystèmes ou des habitats (Alofs et Fowler, 2010). Pour la suite de nos recherches, nous envisageons aussi le recensement des animaux favorisés par la fragmentation dans la zone d'étude. Cet aspect pourrait permettre de mieux comprendre la dynamique des espèces dans les zones d'étude.

L'action anthropique à travers toutes ses formes (abattage des arbres, prélèvement des roches et du sable, désherbage accru, construction anarchique etc..) augmenterait aussi de façon indirecte le taux de mortalité des populations naturelles en dégradant et en compromettant la santé de la zone littorale, notamment à Matombi. Le morcellement de l'écosystème étudié ici pourrait être responsable à moyen et à long terme, de l'appauvrissement des ressources utiles à l'homme, à travers une homogénéisation de la qualité de celles-ci. Consécutif à l'abattage des arbres par l'homme dans les gorges de Diosso et le désherbage accru du littoral, le couvert végétal qui devrait amoindrir par l'action du feuillage, l'énergie cinétique des pluies et empêcher le ruissellement et par conséquent l'érosion, a été détruit. De même, la strate herbacée qui couvre le sol ainsi que la litière et l'humus facilitant l'infiltration de l'eau ont été détruites; Il reste qu'il est difficile, en raison notamment de la pauvreté endémique des populations locales, de véhiculer une politique limitant le déboisement et le désherbage. En effet, l'utilisation du bois de chauffe et de la strate herbacée sous forme de fourrage à des fins domestiques, est nécessaire au fonctionnement optimal des ménages. Par ailleurs, le transfert des matières provenant des gorges de Diosso modifierait la nature de l'eau de la rivière Matombi à travers la variation du $\mathrm{pH}$, de la salinité et même du taux d'oxygène dissous. Cette modification systématique des conditions pourrait être à l'origine des migrations des espèces animales aquatiques et la disparition de la flore environnante. L'action de l'homme sur le littoral de Matombi a conduit à une raréfaction progressive des espèces vivantes, dont le résultat est le remplacement de la variabilité par l'uniformité, la diversité par la dominance et la richesse spécifique par la rareté.

La remontée du niveau de l'océan Atlantique pourrait s'expliquer à travers le réchauffement climatique par l'émission des gaz à effet de serre, notamment le dioxyde de carbone à la suite de l'abattage des arbres et la destruction du couvert végétal. La zone de Matombi soumise au même phénomène, participerait à la modification du climat global. En effet, les résultats obtenus lors d'études précédentes indiquent que les arbres et le couvert végétal, qui auraient dû stocker le dioxyde de carbone, évitant en cela qu'il soit excessivement relâché dans l'atmosphère, ont été détruits (Ramade, 1995).

La mise à nu de la falaise consécutive à la diminution du système racinaire, de la litière et de l'humus, a entraîné une fragilisation du littoral préalablement érodé. Les différents horizons du littoral à Matombi subissent des variations considérables suite à l'avancée du niveau de l'océan et aux effets des eaux de ruissellement. Les matériaux sableux et sablo-argileux offrent ainsi une résistance limitée aux fortes précipitations. Leur présence explique au moins en partie les éboulements et les variations d'horizons dans notre zone d'étude.

La baie de Loango est donc soumise à une série de fragmentation. Celle-ci est d'origine naturelle à travers les érosions dues aux précipitations dont la moyenne est de 1260 $\mathrm{mm}$ /an dans la région de Pointe-Noire, ainsi que les variations du niveau de la mer d'une part, et par les activités anthropiques à travers l'installation des zones touristiques et l'élimination abusive de la strate herbacée et arbustive d'autre part. 


\section{Conclusion}

Les résultats obtenus au cours de ces travaux devraient contribuer à mieux sensibiliser les décideurs mais aussi les populations locales, sur les risques provoqués par un morcellement excessif des écosystèmes en présence: conscientiser sur les risques d'abattage des arbres dans l'accélération des phénomènes d'érosion et l'exploitation excessive des zones fragilisées par la diminution de la strate herbacée. Les fragmentations observées au cours de notre étude ont engendré une diminution des surfaces de milieu favorable avec comme conséquence, la baisse des biodiversités animale et végétale. Une telle situation non maîtrisée pourrait conduire à des menaces de l'entretien des processus écologiques fondamentaux, gage d'un équilibre dynamique des cycles biogène et biologique.

\section{REFERENCES}

Alofs KM, Fowler NL. 2010. Habitat fragmentation caused by woody plant encroachment inhibits the spread of an invasive grass. Journal of Applied Ecology, 47(2): 338-347.

Adams MW. 2004. Against Extinction: the Story of Conservation. Earthscan: London, UK \& Sterling, VA USA; 311p.

Anonyme. 2001. Biodiversité, Bois et Forêt des Tropiques. CIRAD : Montpellier, France; 268 p.

Aron A, Grassé PP. 1966. Précis de Biologie Animale ( $8^{\text {ème }}$ édn). Masson et Cie; 1421 p.

Baker AS. 1999. Mites and Ticks of Domestic Animals. An Identification Guide and Information Source. The stationary Office: London; 240p.

Batchy AC. 2001. La dégradation physique du littoral congolais. Mémoire de Maîtrise en géographie, Univ. Marien NGOUABI, Brazzaville, 127p.

Batchy AC, Locko A. 2003. Rapport du séminaire de sensibilisation et d'information des populations côtières en matière de prévention et de lutte contre les phénomènes d'érosion côtière dans la baie de Loango. Pointe-Noire, 27p.

Batchy AC. 2004. Etude géomorphologique $\mathrm{du}$ littoral congolais, approche méthodologique. Mémoire de DEA, Univ. Marien NGOUABI, Brazzaville, $118 \mathrm{p}$.

Blondel J. 1995. Biogéographie, Approche Ecologique et Evolutive, Masson: France; 297p.

Caspari C, Grossman K, Engel H. 1961. Insectes, Arachnides et Myriapodes. Col. Doc. Hist. Nat. Société Française du Livre: France; 192 p.

Chambon JP. 1993. La mortalité des insectes liée à la circulation des automobiles. Cahier de l'OPIE, 88p.

Chauveau L. 2012. Revue de presse nature. Sciences et Avenir, 785: 32-34.

Dajoz R. 2000. Précis d'Ecologie (7 $7^{\text {ème }}$ édition). Dunod: France; 615p.

Durand P. 1999. L'évolution des plages de l'ouest du golfe de Lyon au XXème siècle. Thèse de doctorat en géographie physique, Université Lumière, Lyon2, $507 p$.

Elenga H, Shwartz D, Vincens A. 1992. Changement climatique et action anthropique sur le littoral congolais au cours de l'holocène. Bull. Soc. Géol., 163 (1): 83-90.

Fischer J, Lindenmayer DB. 2007. Landscape modification and habitat fragmentation: A synthesis. Global Ecology and Biogeography, 16: 265-280.

Frontier S, Pichod-Viale D. 1998. Ecosystèmes: Structure, Fonctionnement, Evolution ( $2^{\text {ème }}$ ed). Dunod: France; $447 \mathrm{p}$.

Grassé PP. 1976. Traité de Zoologie, Anatomie, Systématique, Biologie des Insectes (Tome VIII, Fasc IV). Masson: France; $975 \mathrm{p}$.

Grassé PP. 1996. Zoologie des Vertébrés, ( $3^{\text {ème }}$ édn). Masson: France; 198p.

Kerth G, Melber M. 2009. Species specific barrier effets of motorway on habitat use of two threatement forest - living bat 
species. Biological Conservation, 142(2): 270-279.

Krues A, Tscharntke T. 1994. Habitat fragmentation, species loss and biological control. Science, 1: 581-1 584.

Levejoy TE, Bierregard RO, Rylands AB, Malcom JR, Quintela CE, Harper LH, Brown KS, Powel AH, Powel GNV, Schubar HO, Hays M. 1986. Edge and other effects of isolation on Amazon forests fragmentations. In Conservation Biology: the Science of Scarcity and Diversity, Soulé ME (ed). Sunderland, Sinauer; 257-285.

Makany L. 1063. Contribution à l'étude de la végétation côtière du Congo-Brazzaville. Thèse de $3^{\text {ème }}$ cycle, Orsay, $94 p$.

Makany L. 1964. La côte atlantique du Congo. Peking Symp. Nat. Sci., 2: 891911.

Masieri W. 1996. Statistique et Calcul des Probabilités ( $7^{\text {̀̀me }}$ édn). Editions Dalloz; $369 \mathrm{p}$.

Neuenschwander P, Sinsin B, Goergen G. 2011. Protection de la Nature en Afrique de l'Ouest: Une Liste Rouge pour le Bénin. IITA: Ibadan, Nigeria; 365p.

Picker M, Griffiths C, Weaving A. 2004. Field Guide to Insects of South Africa. Jeanne Hromnik Editor; 444p.
PNUE. 2000. Global Environment 2000. PNUE 320p.

Ramade F. 1995. Eléments d'Ecologie Appliquée ( $5^{\text {ème }}$ éd). Ediscience International: Paris; 586p.

Ramade F. 2002. Dictionnaire Encyclopédique de l'Ecologie et des Sciences de l'Environnement (2 ${ }^{\mathrm{ème}}$ édn). Dunod: France; 1075p.

Ramade F. 2005. Eléments d'Ecologie. Ecologie Appliquée (6 $6^{\text {ème }}$ éd). Dunod: France; 864p.

Sitou L. 1994. Les cirques d'érosion dans la région de Pointe-Noire (Congo). Etude géomorphologique. Thèse de doctorat, Univ. Louis Pasteur, Strasbourg I, 224p.

Souza OF, Brown VK. 1994. Effects of habitat fragmentation on Amazonian termite communities. Journal of Tropical Ecology, 10: 197-206.

Thomas CD, Cameron A, Green RE. 2004. Extinction risk from climate changes. Nature, 427: 145-148.

Wood B, Gillman MP. 1998. The effects of disturbance on forest butterflies using two methods of sampling in Trinidad. Biodiversity and Conservation, 7: 597-61. 